\title{
$\mathrm{G}$ wie die Geschichte eines Scheiterns oder la grande illusion
}

\section{Dominik Heim}

PD Dr. med., Klinik Hohmad, Thun, Mitglied FMH

Ich glaube, es war Rick Wakeman, der langjährige Keyboarder der Progressive-Rock-Gruppe Yes, der einmal auf einem seiner Soloalben sagte: "There was a time I did believe the stories I was told.» Mit zunehmendem Alter kam dann offensichtlich eine gewisse Skepsis. Gewisse Geschichten sind aber wahr. Dann heisst es im Filmtrailer «based on a true story». Und um der wahren Geschichte etwas ihre Vehemenz zu nehmen, liest man dann in der Belletristik: "Ähnlichkeiten mit lebenden Personen sind rein zufällig.» Denn die Gefahr, dass sich Leser in einer Romanfigur erkennen (wollen), ist schliesslich da. Und «Nestbeschmutzer», heute whistleblower genannt, kippt man gerne aus dem Nest: Meinrad Inglin, einer der ganz wichtigen Schweizer Autoren des 20. Jahrhunderts, trieben die Steinwürfe von aufgebrachten Schwyzer Bürgern nach seinem Buch Die Welt in Ingoldau (1922) ins Zürcher Exil. Er war ein sehr genauer Chronist seiner Zeit, halt ein kritischer Zeitgeist, der den bürgerlichen Filz und die katholische Kirche schonungslos blosstellte. Und Ingoldau war eben das Städtchen Schwyz. Seine Geschichte von damals könnte in vielem auch heute noch stimmen:

Und so kam es, dass sich eine Gemeinde Sorgen machte, was denn mit ihrem Tal geschehen würde, wenn sich die dortigen Landärzte einmal altershalber zurückziehen würden. Die dann folgenden politisch-medizinischen Gespräche mit den ansässigen Ärzten verliefen aus nicht genau bekannten Gründen im Sand, wovon es in diesem Tal wenig gab. Und auch der Schnee blieb je länger, je mehr aus, und doch war der lokale Tourismus eine wichtige Geldquelle, und damit war auch die medizinische Versorgung ein berechtigtes Anliegen. Man suchte deshalb weiter und fand eine medizinische Organisation, die (unter bestimmten Prämissen) willig war, sich in diesem Tal mit einer Walk-in-Praxis mit mehreren Ärzten zu engagieren. Das entsprach ja auch etwas dem neuen Bedürfnis der Patienten, schnell, ohne Anmeldung und ganz einfach zu einer medizinischen Notfalluntersuchung zu kommen. Sie sollte kompetent sein, bildgebend umfassend und sich eventuell noch mit einem Shopping-Termin verbinden lassen [1]. Es kam nicht gut an. An der Gemeindeversammlung wurde der Gemeinde das Recht zu einer Subventionierung dieser neuen Praxis abgesprochen. Wüste Zeitungsartikel erschienen in der lokalen Presse, die angesprochene Vehemenz blühte. Die Praxis polarisierte: Man ging extra in diese neue Praxis (und erzählte Geschichten) oder eben gerade extra nicht. Der medizinischen Organisation ging dann das Geld aus (sagt man), und sie zog sich zurück. Und alles war wie früher ...

Gründe für das Scheitern wie nicht eingehaltene Versprechen, politisches Versagen oder «Futterneid» der hiesigen Ärzte wurden dann kolportiert. Wahrscheinlich stimmt ja von allem etwas. Die Tatsache aber ist, dass dieser Versuch fehlschlug, eine medizinische Grundversorgung in dem Tal auch in Zukunft zu garantieren. Was in einer Stadt funktioniert, muss kein Erfolgsrezept auch in der Peripherie sein. Kommt Ihnen da auch ein Filmregisseur in den Sinn? Nein? Jean Renoir habe ich eigentlich damit nicht gemeint ... oder doch?

Jean Renoir, der französische Regisseur, drehte 1937 La Grande Illusion, eine Geschichte von Kriegsgefangenen unterschiedlicher Gesellschaftsschichten im Ersten Weltkrieg. Er war der Ansicht, dass Nationalismus, Klassen- und Rassenunterschiede und Krieg die gesellschaftlichen Probleme nicht lösen können. Doch der Zweite Weltkrieg fand trotzdem statt. Und jetzt «la grande illusion» auch hier: Die Chance ist vertan, eine Lösung gab es nicht. Die Rechnung zahlen die Patienten! Mit ihrem Ohrenweh werden sie weiterhin 3 Tage bis zum Arztbesuch warten müssen (sagen sie), und wenn sie sich inkompetent behandelt fühlen (sagen sie), dann müssen sie zum Tal hinaus. Und ich muss jetzt vielleicht ins Exil!

\footnotetext{
Literatur

1 Oertle D. Die Hausärzte sterben aus: na und? Schweiz Ärztezeitung. 2016;97(51-52):1804-5.
} 